\title{
Similarity theory based optimization model of function chain design
}

\author{
solution \\ Yu-yun Kang \\ College of Mechanical Engineering, Linyi University, Linyi 276005, China \\ kyygood@163.com
}

\begin{abstract}
Keywords: Conceptual design, Similarity theory, Generalized Distance, Ant Colony Optimization. Abstract. A mathematical model is proposed to analyse the optimization method of function chain design solution. In the approach, similarity theory is used to analyse and calculate the Generalized Distance (GD) of two adjacent components in a design solution. The evaluation criteria of sub-functions are used to select alternative components. Under the premise of regarding the Generalized Distance as the path passed through by ants, Ant Colony Optimization (ACO) is used to identify appropriate solutions automatically. Evaluation of single component and compatibility of two adjacent components are considered in a design solution by the proposed optimization model.
\end{abstract}

\section{Introduction}

Product concept evaluation in a product development has been identified as the major activities needed for obtaining an optimal design solution. Techniques are still required to generate design solutions and identify appropriate solutions automatically from many candidate solutions. A optimization model is proposed in this paper to develop a design scheme based on similarity theory and ACO for product conceptual design evaluation. The specific focus of this research is the combinatorial optimization model of design solutions.

\section{Similarity theory}

Similarity theory [1-3] is a systematic methodology that utilizes a suit of approaches to measure comparability between two things. It considers that the system is composed of sub-systems or elements, and that the element is composed of some characteristics which have the weighed value.

It is supposed that there are two components (or sub-systems) A and B. As for a similar characteristic $w$ of $\mathrm{A}$ and $\mathrm{B}$, the weighed value is $\gamma_{w}(A)$ and $\gamma_{w}(B)$ respectively. The value of $\eta_{w}$ which reflects the similarity degree of characteristic $w$ is calculated by Equation1:

$\eta_{w}=\frac{\min \left\{\gamma_{w}(A), \gamma_{w}(B)\right\}}{\max \left\{\gamma_{w}(A), \gamma_{w}(B)\right\}}$.

It is supposed that $k$ is the number of characteristics of component $\mathrm{A}$, and $l$ is the number of characteristics of component B, and $m$ is the number of same characteristics between A and B. $q_{1}$ is employed to reflect the similarity degree of similar characteristics to all characteristics. The value of $q_{1}$ is calculated by following Equation: 
$q_{1}=\frac{m}{r+l-m}$.

$\eta_{w}$ is the similarity degree of characteristic $w . d_{w}$ is the weighting coefficient of the similar characteristic $w . q_{2}$ is employed to reflect the similarity degree of all similar characteristics. Its value is calculated by following Equation:

$q_{2}=\sum_{w=1}^{m} d_{w} \eta_{w}$

If $Q$ is employed to reflect the similarity degree of components $\mathrm{A}$ and $\mathrm{B}$, its value is calculated by following Equation:

$Q=q_{1} \cdot q_{2}=\frac{m}{k+l-m} \sum_{w=1}^{m} d_{w} \eta_{w}$.

The range of $Q$ is $\{0,1\}$. If the value of $Q$ is bigger, the similarity of components $\mathrm{A}$ and $\mathrm{B}$ is higher, and the components $\mathrm{A}$ and $\mathrm{B}$ are easier to combine together.

\section{Generalized Distance}

As mentioned in above section, $Q$ is the similarity degree of two components or sub-systems, and its value range is $\{0,1\}$. If the value of $Q$ is bigger, two components or sub-systems are more similar. A parameter $D$ named Generalized Distance (GD) is defined as a contrary meaning for $Q$. Its value is calculated by Equation 5: $D=1-Q+C$

in which $C$ is a constant, and the value is provided according to the design requirement. $D$ is used to indicate the compatibility of adjacent components. If its value is smaller, the compatibility of adjacent components is better.

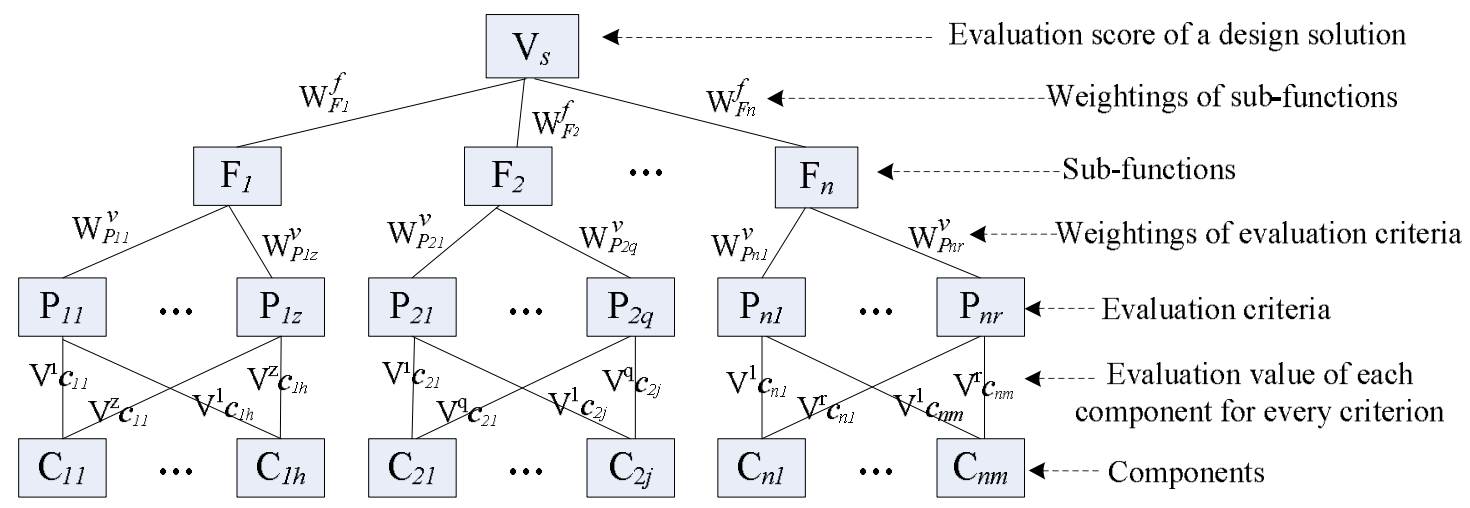

Fig. 1. Evaluation tree. 


\section{Evaluation criteria and weightings}

As shown in Fig. 1, designer's requirements are viewed as components' evaluation criteria and weightings of evaluation criteria and sub-functions. These are prerequisite for automatically identifying optimal design solutions. The sum of weightings of all evaluation criteria for a sub-function is 1 . For each evaluation criterion (e.g. $P_{11}$ ) of a sub-function (e.g. $\left.F_{1}\right)$, there are different evaluation values (e.g. $V_{C 11}^{1}, V_{C 1 h}^{1}$ ) for different components (e.g. $C_{11}, C_{1 h}$ ). Computationally, the component's evaluation score for a sub-function is calculated by adding the evaluation values multiplied by weighting of relative criterion. For example, there is a design solution $X$ and it was composed of components: $C_{11}, C_{2 j}, \ldots, C_{n m}$. Its evaluation score can be calculated by Equation 9.

$$
\begin{aligned}
V_{s}^{X}= & \left(V_{C_{11}}^{1} \cdot W_{P_{11}}^{v}+V_{C_{11}}^{2} \cdot W_{P_{12}}^{v}+\mathrm{L}+V_{C_{11}}^{z} \cdot W_{P_{12}}^{v}\right) \cdot W_{F 1}^{f}+\left(V_{C_{2 j}}^{1} \cdot W_{P_{21}}^{v}+V_{C_{2 j}}^{2} \cdot W_{P_{22}}^{v}+\mathrm{L}+V_{C_{2 j}}^{q} \cdot W_{P_{2 q}}^{v}\right) \cdot W_{F 2}^{f} \\
& +\mathrm{L}+\left(V_{C_{n n}}^{1} \cdot W_{P_{n 1}}^{v}+V_{C_{n n}}^{2} \cdot W_{P_{n 1}}^{v}+\mathrm{L}+V_{C_{n n}}^{r} \cdot W_{P_{n n}}^{v}\right) \cdot W_{F n}^{f}
\end{aligned}
$$

where $V_{s}^{X}$ is the evaluation score of the design solution $X$. The meaning of other parameters is shown in Figure 1.

\section{Optimization model with ACO}

Two factors are considered in the conceptual design optimization process: one is the evaluation score of an alternative component, and the other is GD of adjacent components. The evaluation score of a component corresponding to each sub-function is calculated according to the following Equation:

$$
e\left(C_{i j}\right)=\sum_{k=1}^{m} W_{k} f_{i j}\left(V_{k}\right)
$$

where $e\left(C_{i j}\right)$ is the evaluation score of the component $j$ for the sub-function $i . m$ is the amount of evaluation criteria for the sub-function $i, k$ is a evaluation criterion for sub-function $i$. $W_{k}$ is the weighting of criterion $k . f_{i j}\left(V_{k}\right)$ is the function used to acquire evaluation value of component $C_{i j}$ for evaluation criterion $k$. According to Equation 11, Equation 9 can be simplified as the following Equation:

$$
V_{s}^{X}=\sum_{i=1}^{n} W_{F i} e\left(C_{i j}\right)=\sum_{i=1}^{n} W_{F i} \sum_{k=1}^{m} W_{k} f_{i j}\left(V_{k}\right)
$$

where $n$ is the amount of sub-functions, and $W_{F i}$ is the weighting of the sub-function $i$.

Under the comprehensive consideration of the GD and the evaluation score of the components, the evaluation score of a design solution may be calculated by the following Equation:

$$
E=\sum_{i=1}^{n} W_{F i} e\left(C_{i j}\right)+\xi \sum_{i=1}^{n} \frac{1}{D_{l j}}
$$


where $E$ is the evaluation score of a design solution, $\xi$ is a balance coefficient which makes the evaluation score of components and the value of GD in the same magnitude, $D_{l j}$ is the GD of two adjacent components, $l$ and $j$ represent alternative component respectively for sub-function $i-1$ and $i$. Using Equation 9, the goal of product design is transformed to calculating the maximum value of $E$. The optimization process with ACO is as follows:

Step 1: Initialization

Step 2: Iteration process of the optimization program

While not $\left[E^{g}(t)-E^{g}(t-1)\right] \leq \varepsilon$ do

(1) For $k=1$ to $m$ ( $m$ is the population of ants)

For $i=0$ to $n$ ( $n$ is the amount of sub-functions)

Ant $k$ selects path according to the probability calculated.

End for $i$

The evaluation score of $E_{k}$ is acquired by Equation 9 and compared with the score of $E_{k-1}$, then the bigger is recorded.

End for $k$

(2) After a cycle is accomplished, the biggest score $E^{g}(t)$ of the cycle is acquired from $E_{1}$ to $E_{k}$ and correspondingly the best route of the cycle is identified.

(3) Pheromone $\tau_{i j}(t)$ of each path is renewed.

End for while.

Step 3: The biggest evaluation score of each cycle is acquired and compared with each other resulting in the optimal evaluation score of all cycles is selected out and the best route is identifyed.

Step 4: End of program.

\section{Conclusion}

An evaluation model with similarity theory and ACO is introduced in this paper. Compared to traditional concept evaluation model, evaluation of single component and compatibility of two adjacent components are all considered.

\section{Acknowledgments}

This research was financially supported by Linyi University Doctor Research Grants numbered LYDX2015BS014.

\section{Reference}

[1] Zhao, Y., Xu, K.Z., Zha, J.Z., 2007. Mechanical transmission scheme optimal design based on a new ant algorithm (in Chinese). Computer Integrated Manufacturing Systems, 13(5), 925-930. 
[2] Zhou, M.L., 1999. Formation Principles of Similarity between Similar Systems. International Journal of General System, 27(6), 495-504.

[3] Zhou, M.L., 2004. Similarity and Complexity in Unitization System Integrated Design (in Chinese). China Mechanical Engineering, 15(7), 604-607. 